\title{
SOLVING MULTI-LEVEL MULTIOBJECTIVE FRACTIONAL PROGRAMMING PROBLEM WITH ROUGH INTERVAL PARAMETER IN NEUTROSOPHIC ENVIRONMENT
}

\author{
Firoz Ahmad ${ }^{1,2} \odot$, Shafiq Ahmad ${ }^{3, *}$, Ahmed T. Soliman ${ }^{3}$ And Mali Abdollahian ${ }^{4}$
}

\begin{abstract}
In this study, a novel algorithm is developed to solve the multi-level multiobjective fractional programming problems, using the idea of a neutrosophic fuzzy set. The co-efficients in each objective functions is assumed to be rough intervals. Furthermore, the objective functions are transformed into two sub-problems based on lower and upper approximation intervals. The marginal evaluation of pre-determined neutrosophic fuzzy goals for all objective functions at each level is achieved by different membership functions, such as truth, indeterminacy/neutral, and falsity degrees in neutrosophic uncertainty. In addition, the neutrosophic fuzzy goal programming algorithm is proposed to attain the highest degrees of each marginal evaluation goals by reducing their deviational variables and consequently obtain the optimal solution for all the decision-makers at all levels. To verify and validate the proposed neutrosophic fuzzy goal programming techniques, a numerical example is adressed in a hierarchical decision-making environment along with the conclusions.
\end{abstract}

Mathematics Subject Classification. 03B52, 03F55, 62J05, 62J99.

Received June 21, 2020. Accepted July 21, 2021.

\section{INTRODUCTION}

Most often, the mathematical programming problems consist of only one decision-maker who takes the decisions all alone. Apart from that, many decision-making problems involve hierarchical decision structures, each with independent, and most often contradictory. Such decision-making scenarios are termed as decentralized planning problems. Thus the hierarchical decision-making texture of the problem is formulated as multi-level programming problems (MLPPs). If there are only two decision-makers, then it becomes bi-level programming problems, tri-level for three decision-makers, and so on. The fundamental concepts behind the MLPPs optimization techniques are that the leader-level decision-maker define his/her goals/target and then seeks the optimal solution from each sub-ordinate level of the organization that has calculated individually. The follower-level decisions are later submitted and satisfied by the leader-level because of the organization's overall benefit. There

Keywords. Rough intervals, Indeterminacy membership function, Neutrosophic goal programming algorithm, Hierarchical decision-making problems.

1 Department of Statistics and Operations Research, Aligarh Muslim University, Aligarh, India

2 Indian Statistical Institute, Bangalore Centre, 8th Mile, Mysore Road, Bangalore 560059, India

3 Industrial Engineering Department, College of Engineering, King Saud University, P.O. Box 800, Riyadh 11421, Saudi Arabia

4 School of Science, College of Sciences, Technology, Engineering, Mathematics, RMIT University, GPO Box 2476,

Melbourne, Victoria 3001, Australia.

*Corresponding author: ashafiq@ksu.edu.sa 
may be more than one fractional objective function that is to be optimized by different levels in MLPPs. Such kind of decentralized decision-making problems is termed as multi-level multiobjective fractional programming problems (ML-MOFPPs).

Firstly, the concept of rough set theory was introduced by [25] in 1982 as a technique for the collaborative significance of vagueness and uncertainty. Most often, the rough set theory is highlighted to be a prominent mathematical device for the analysis and interpretation of a vague knowledges of things called actions in decision problems [26]. It has been efficiently implemented in many real-life problems including decision sciences, pharmacology, civil engineering and management sciences [14] etc. Recently, rough linear programming is proposed by $[16,23,24]$ and they introduced two solutions concepts as surely interval and possibly range for the existance of optimal solution. Many researchers such as $[3,4,17,19,20,28]$ have worked on intuitionistic fuzzy and neutrosophic research domain. The rough set theory is based on fundamental logic that any uncertain quantification of any objects can be depicted by a pair of specified interval known as the lower and upper approximation of the uncertain quantification. To any ambiguous knowledge $V$, a lower approximation is restrained of entire element that surely associated with the vague knowledge $V$ and an upper approximation is restrained of entire element that possibly associated with the ambiguous knowledge $V$. More precisely, the lower approximation is regarded as the union of all elementary ideas that are incorporated in the vague knowledges $V$, whereas the union of all primary ideas having non-empty intersection with the vague knowledges $V$ is identified by the upper approximation interval respectively.

Many research articles in the domain of ML-MOFPPs utilized either fuzzy or intuitionistic fuzzy based optimization method that either inherently consider the membership and non-membership functions of the object into a feasible set. But it may be possible to arise indeterminacy degree while making decisions. Such kind of issue cannot be dealt with fuzzy and intuitionistic fuzzy based optimization methods. Thus, the extensions or generalizations of fuzzy set (FS) and intuitionistic fuzzy set (IFS) has been introduced by incorporating indeterminacy degree and named as neutrosophic set. Smarandache [31] proposed the idea of neutrosophic set (NS). The word "neutrosophic" conntains two different words, "neutre" from French means, neutral, and "sophia" from Greek means, skill/wisdom, aving meaning "knowledge of neutral thoughts" and differ from FS and IFS (see [5-7,31]). The neutral/indeterminacy ideas of neutrosophic set explores the future research direction in the real-life application. Many researchers such as $[5,7,9]$ and $[11,12]$ made a contribution in the domain of neutrosophic optimization techniques along with real-life applications.

The primary aim and objective of the study is to furnish the more realistic framework for ML-MOFPPs to determine the better solution results under rough intervals. Moreover, the proposed approach has an advantage that it does not only consider the maximization and minimization of the DM's satisfaction and dis-satisfaction level but also optimize the indeterminacy/neutral degree of satisfaction. In this paper, the neutrosophic fuzzy goal programming (NFGP) algorithm is introduced to solve the multi-level multiobjective fractional programming problems. The propounded NFGP procedures is based on neutrosophic fuzzy decision set and applied to ML-MOFPPs. To formulate proposed NFGP models of the ML-MOFPPs, neutrosophic goals of the objectives are obtained by individual optimal solutions. The associated membership functions is then depicted for marginal evaluations of each objective function under the neutrosophic environment. These marginal evluations are converted into neutrosophic flexible membership goals by employing over and under deviational variables and allowing highest truth membership value (unity), indeterminacy value (half), and a falsity value (zero) as aspiration levels to each of them. To determine the membership functions of the constraints monitored by any level decision-maker, the optimal solution of the corresponding MOFPP is separately solved. A marginal relaxation of the decisions is prescribed to avoid decision deadlock. The proposed NFGP solution algorithm provides an extension of the work presented by $[1,18,21]$ and [24] under neutrosophic environment which deals with multi-level multiobjective fractional programming problems. It also extend the work of [27] by introducing the NFGP algorithm to MLPPs with a multiple fractional objective at various level. Ultimately, the final model entertains the marginal evaluations for the described neutrosophic goals of the objective functions and the constraints at all levels which are determined separately for each level except the follower. 


\section{Preliminaries}

In this section, some basic definitions related to rough intervals and neutrosophic set is discussed.

Definition 2.1 ([16]). (Rough Interval (RI)) Any qualitative value $V$ is said to be a rough interval if and only iff one can assign two closed intervals $V_{*}$ and $V^{*}$ on $\mathbb{R}$ to it where $V_{*} \subseteq V^{*}$. Furthermore,

1. If $y \in V_{*}$ then $V$ definitely takes $y$ (depicted as $y \in V$ ).

2. If $y \in V^{*}$ then $V$ probably takes $y$.

3. If $y \notin V^{*}$ then $V$ surely does not take $y$ (depicted as $y \notin V$ ).

$V_{*}$ and $V^{*}$ are known as the lower approximation interval (LAI) and the upper approximation interval (UAI) of $V$, respectively. Moreover, $V$ is represented by $V=\left(V_{*}, V^{*}\right)$. It should be noted that the intervals $V_{*}$ and $V^{*}$ are not complement of each other.

Definition $2.2([16])$. (Arithmetic Operations on RIs) The arithmetic operations on RIs fully depends on interval arithmetic. Now, some of the well-known arithmetic operations can be presented as follows:

Let $V=\left(\left[\underline{v}^{L}, \underline{v}^{U}\right],\left[\bar{v}^{L}, \bar{v}^{U}\right]\right)$ and $W=\left(\left[\underline{w}^{L}, \underline{w}^{U}\right],\left[\bar{w}^{L}, \bar{w}^{U}\right]\right)$ be two RIs. Then, we have

1. (Addition): $V+W=\left(\left[\underline{v}^{L}+\underline{w}^{L}, \underline{v}^{U}+\underline{w}^{U}\right],\left[\bar{v}^{L}+\bar{w}^{L}, \bar{v}^{U}+\bar{w}^{U}\right]\right)$.

2. (Subtraction): $V-W=\left(\left[\underline{v}^{L}-\underline{w}^{U}, \underline{v}^{U}-\underline{w}^{L}\right],\left[\bar{v}^{L}-\bar{w}^{U}, \bar{v}^{U}-\bar{w}^{L}\right]\right)$.

3. (Negation): $-V=\left(\left[-\underline{v}^{L},-\underline{v}^{U}\right],\left[-\bar{v}^{L},-\bar{v}^{U}\right]\right)$.

4. (Union): $V \cup W=\left(\left[\min \left(\underline{v}^{\bar{L}}, \underline{w}^{L}\right), \max \left(\underline{v}^{U}, \underline{w}^{U}\right)\right],\left[\min \left(\bar{v}^{L}, \bar{w}^{L}\right), \max \left(\bar{v}^{U}, \bar{w}^{U}\right)\right]\right)$.

5. (Intersection): $V \cap W=\left(\left[\max \left(\underline{v}^{L}, \underline{w}^{L}\right), \min \left(\underline{v}^{U}, \underline{w}^{U}\right)\right],\left[\max \left(\bar{v}^{L}, \bar{w}^{L}\right), \min \left(\bar{v}^{U}, \bar{w}^{U}\right)\right]\right)$.

Remark 2.3. Using the properties of RIs, we have

$$
\left[\underline{v}^{L}, \underline{v}^{U}\right] \subseteq\left[\bar{v}^{L}, \bar{v}^{U}\right] \rightarrow \bar{v}^{L} \leq \underline{v}^{L} \leq \underline{v}^{U} \leq \bar{v}^{U}
$$

Definition 2.4 ([8]). Suppose a universal discourse $Y$ such that $y \in Y$, then a neutrosophic set $A$ in $Y$ can be depicted by truth $\mu_{A}(y)$, indeterminacy $\lambda_{A}(y)$ and a falsity $\nu_{A}(y)$ membership functions in the following form:

$$
A=\left\{<y, \mu_{A}(y), \lambda_{A}(y), \nu_{A}(y)>\mid y \in Y\right\}
$$

where $\mu_{A}(y), \lambda_{A}(y)$ and $\nu_{A}(y)$ are real standard or non-standard subsets belong to $] 0^{-}, 1^{+}$, also given as, $\left.\mu_{A}(y): Y \rightarrow\right] 0^{-}, 1^{+}\left[, \lambda_{A}(y): Y \rightarrow\right] 0^{-}, 1^{+}\left[\right.$, and $\left.\nu_{A}(y): Y \rightarrow\right] 0^{-}, 1^{+}$. There is no restriction on the sum of $\mu_{A}(y), \lambda_{A}(y)$ and $\nu_{A}(y)$, so we have

$$
0^{-} \leq \sup \mu_{A}(y)+\lambda_{A}(y)+\sup \nu_{A}(y) \leq 3^{+}
$$

Definition 2.5 ([8]). A single valued neutrosophic set $A$ over universe of discourse $Y$ is defined as

$$
A=\left\{<y, \mu_{A}(y), \lambda_{A}(y), \nu_{A}(y)>\mid y \in Y\right\}
$$

where $\mu_{A}(y), \lambda_{A}(y)$ and $\nu_{A}(y) \in[0,1]$ and; $0 \leq \mu_{A}(y)+\lambda_{A}(y)+\nu_{A}(y) \leq 3$ for each $y \in Y$.

Definition 2.6 ([8]). The complement of a single valued neutrosophic set $A$ is represented as $c(A)$ and defined by $\mu_{c(A)}(y)=\nu_{A}(y), \lambda_{c(A)}(y)=1-\nu_{A}(y)$ and $\nu_{c(A)}(y)=\mu_{A}(y)$ respectively. 


\section{Problem Formulation}

Assume that a $t$-level multiobjetive programming problems with maximization-type fractional objective functions at different level. Consider that $D M_{i}$ represents the $i$-th level decision-maker and control over the decision variable $\mathbf{y}_{i}=\left(y_{i 1}, y_{i 2}, \ldots, y_{i n_{i}}\right) \in \mathbb{R}^{\mathrm{n}_{\mathrm{i}}}$ for all $i=1,2, \ldots, t$. Where $\mathbf{y}=\left(\mathbf{y}_{1}, \mathbf{y}_{2}, \ldots, \mathbf{y}_{t}\right) \in R^{n}$ such that $n=n_{1}+{ }_{2}+\ldots+n_{t}$. Furthermore, we assume that

$$
O_{i}(\mathbf{y})=O_{i}\left(\mathbf{y}_{1}, \mathbf{y}_{2}, \ldots, \mathbf{y}_{t}\right): \mathbb{R}^{\mathrm{n}_{1}} \times \mathbb{R}^{\mathrm{n}_{2}} \times \cdots \times \mathbb{R}^{\mathrm{n}_{\mathrm{t}}} \rightarrow \mathbb{R}^{\mathrm{m}_{\mathrm{i}}}, \quad \forall \mathrm{i}=1,2, \ldots, \mathrm{t}
$$

represents the vector-set of a well-defined fractional objective functions to the $i$-th decision makers, $i=1,2, \ldots, t$. The equivalent mathematical expressions for the ML-MOFPP with rough intervals under maximization-type objectives can be stated as follows:

$$
\begin{aligned}
& \text { [1st level] } \\
& \operatorname{Max}_{y_{1}} O_{1}(\mathbf{y})=\operatorname{Max}_{y_{1}}\left(o_{11}(\mathbf{y}), o_{12}(\mathbf{y}), \ldots, o_{1 m_{1}}(\mathbf{y})\right) \\
& \text { where } \mathbf{y}_{2}, \mathbf{y}_{3}, \ldots, \mathbf{y}_{t} \text { solves } \\
& \text { [2nd level] } \\
& \operatorname{Max}_{y_{2}} O_{2}(\mathbf{y})=\operatorname{Max}_{y_{2}}\left(o_{21}(\mathbf{y}), o_{22}(\mathbf{y}), \ldots, o_{2 m_{2}}(\mathbf{y})\right) \\
& \text {... } \\
& \text { where } \mathbf{y}_{t} \text { solves } \\
& {[t-\text { th level }]} \\
& \operatorname{Max}_{y_{t}} O_{t}(\mathbf{y})=\operatorname{Max}_{y_{t}}\left(o_{t 1}(\mathbf{y}), o_{t 2}(\mathbf{y}), \ldots, o_{t m_{t}}(\mathbf{y})\right)
\end{aligned}
$$

subject to

$$
\mathbf{y} \in \mathbf{S}=\left\{\mathbf{y} \in \mathbb{R}^{\mathrm{n}} \mid \mathrm{G}_{1} \mathrm{y}_{1}+\mathrm{G}_{1} \mathrm{y}_{1}+\cdots+\mathrm{G}_{\mathrm{t}} \mathrm{y}_{\mathrm{t}}(\leq \text { or }=\text { or } \geq) \mathbf{q}, \mathbf{y} \geq 0, \mathbf{q} \in \mathbb{R}^{\mathrm{m}}\right\} \neq \phi
$$

where

$$
o_{i j}(\mathbf{y})=\frac{N_{i j}(y)}{D_{i j}(y)}=\frac{\sum_{j=1}^{m_{i}}\left(\left[\underline{c}_{i j}^{L}, \underline{c}_{i j}^{U}\right],\left[\bar{c}_{i j}^{L}, \bar{c}_{i j}^{U}\right]\right) y_{j}+\left(\left[\underline{v}_{i j}^{L}, \underline{v}_{i j}^{U}\right],\left[\bar{v}_{i j}^{L}, \bar{v}_{i j}^{U}\right]\right)}{\sum_{j=1}^{m_{i}} d_{i j} y_{j}+w_{i j}}
$$

such that $\left(\left[\underline{\underline{c}}_{i j}^{L}, \underline{c}_{i j}^{U}\right],\left[\bar{c}_{i j}^{L}, \bar{c}_{i j}^{U}\right]\right)$ are the rough intervals co-efficients of the objective function, $\left(\left[\underline{v}_{i j}^{L}, \underline{v}_{i j}^{U}\right],\left[\bar{v}_{i j}^{L}, \bar{v}_{i j}^{U}\right]\right)$ denotes the rough intervals constants of the numerator part. Further, $\mathbf{S}$ is the multi-level convex constraints in feasible decision set under multi-level multiobjective programming problems. It is assumed that $D_{i j}(y)>0 \forall y \in$ $\mathbf{S}$, also $w_{i j}$ are constants of denominator. The notation $m_{i}, i=1,2, \ldots, t$ denotes the number of objective function under $i$-th decision maker, $m$ is the number of constraints, $c_{k}^{i j}=\left(c_{k 1}^{i j}, c_{k 2}^{i j}, \cdots, c_{k n_{k}}^{i j}\right), k=1,2, \ldots, t, c_{k n_{k}}^{i j}$ are constants and the co-efficients matrices of size $m \times n_{i}$ are depicted as $G_{i}, \forall i=1,2, \ldots, t$.

\subsection{Transformation of ML-MOFPP with rough co-efficients into upper and lower approximations}

The transformation process of ML-MOFPPs with rough coefficient in objective functions into upper and lower approximations is quite tough job in many cases, but conversion phenomenon takes the advantages of Remark 2.3. The equivalent problems of the ML-MOFPP with rough coefficients in objective functions with the aid of specified intervals can be represented as follows:

The surely optimal range of ML-MOFPP (3.2) can be obtained by solving the following two classical LFPPs:

After solving the above classical four ML-MOFPPs simultaneously, the neutrosophic fuzzy goal programming algorithm will be applied. 
The lower approximated intervals in the objective functions (LAI)

\begin{tabular}{|c|c|}
\hline FPP-I & FPP-II \\
\hline [1st level] & {$[1$ st level $]$} \\
\hline $\begin{array}{l}\underset{y_{1}}{\operatorname{Max}} O_{1}(\mathbf{y})=\underset{y_{1}}{\operatorname{Max}}\left(o_{11}(\mathbf{y}), o_{12}(\mathbf{y}), \ldots, o_{1 m_{1}}(\mathbf{y})\right) \\
\text { where } \mathbf{y}_{2}, \mathbf{y}_{3}, \ldots, \mathbf{y}_{t} \text { solves } \\
{[2 \text { st level] }}\end{array}$ & $\begin{array}{c}\operatorname{Max}_{y_{1}} O_{1}(\mathbf{y})=\operatorname{Max}_{y_{1}}\left(o_{11}(\mathbf{y}), o_{12}(\mathbf{y}), \ldots, o_{1 m_{1}}(\mathbf{y})\right) \\
\text { where } \mathbf{y}_{2}, \mathbf{y}_{3}, \ldots, \mathbf{y}_{t} \text { solves } \\
{[2 \text { st level }]}\end{array}$ \\
\hline $\begin{array}{l}\underset{y_{2}}{\operatorname{Max}} O_{2}(\mathbf{y})=\underset{y_{2}}{\operatorname{Max}}\left(o_{21}(\mathbf{y}), o_{22}(\mathbf{y}), \ldots, o_{2 m_{2}}(\mathbf{y})\right) \\
\text { where } \mathbf{y}_{t} \text { solves } \\
{[t-\text { th level }]}\end{array}$ & $\begin{array}{c}\operatorname{Max}_{y_{2}} O_{2}(\mathbf{y})=\operatorname{Max}_{y_{2}}\left(o_{21}(\mathbf{y}), o_{22}(\mathbf{y}), \ldots, o_{2 m_{2}}(\mathbf{y})\right) \\
\text { where } \mathbf{y}_{t} \text { solves } \\
{[t-\text { th level }]}\end{array}$ \\
\hline $\begin{array}{l}\underset{y_{t}}{\operatorname{Max}} O_{t}(\mathbf{y})=\operatorname{Max}_{y_{t}}\left(o_{t 1}(\mathbf{y}), o_{t 2}(\mathbf{y}), \ldots, o_{t m_{t}}(\mathbf{y})\right) \\
\text { subject to }\end{array}$ & $\begin{array}{c}\operatorname{Max}_{y_{t}} O_{t}(\mathbf{y})=\operatorname{Max}_{y_{t}}\left(o_{t 1}(\mathbf{y}), o_{t 2}(\mathbf{y}), \ldots, o_{t m_{t}}(\mathbf{y})\right) \\
\text { subject to }\end{array}$ \\
\hline $\begin{array}{l}\mathbf{y} \in \mathbb{R}^{\mathrm{n}} \mid \mathrm{G}_{1} \mathrm{y}_{1}+\mathrm{G}_{1} \mathrm{y}_{1}+\cdots+\mathrm{G}_{\mathrm{t}} \mathrm{y}_{\mathrm{t}}(\leq \text { or }=\text { or } \geq) \mathbf{q} \\
\mathbf{y} \geq 0, \mathbf{q} \in \mathbb{R}^{\mathrm{m}} \neq \phi \\
\text { where }\end{array}$ & $\begin{array}{c}\mathbf{y} \in \mathbb{R}^{\mathrm{n}} \mid \mathrm{G}_{1} \mathrm{y}_{1}+\mathrm{G}_{1} \mathrm{y}_{1}+\cdots+\mathrm{G}_{\mathrm{t}} \mathrm{y}_{\mathrm{t}}(\leq \text { or }=\text { or } \geq) \mathbf{q} \\
\mathbf{y} \geq 0, \mathbf{q} \in \mathbb{R}^{\mathrm{m}} \neq \phi \\
\text { where }\end{array}$ \\
\hline$o_{i j}(\mathbf{y})=\frac{N_{i j}(y)}{D_{i j}(y)}=\frac{\sum_{j=1}^{m_{i}} \underline{c}_{i j}^{L}+\underline{v}_{i j}^{L}}{\sum_{j=1}^{m_{i}} d_{i j} y_{j}+w_{i j}}, i=1,2, \cdots, t$ & $o_{i j}(\mathbf{y})=\frac{N_{i j}(y)}{D_{i j}(y)}=\frac{\sum_{j=1}^{m_{i}} \underline{c}_{i j}^{U}+\underline{v}_{i j}^{U}}{\sum_{j=1}^{m_{i}} d_{i j} y_{j}+w_{i j}}, i=1,2, \cdots, t$. \\
\hline
\end{tabular}

The upper approximated intervals in the objective functions (UAI)

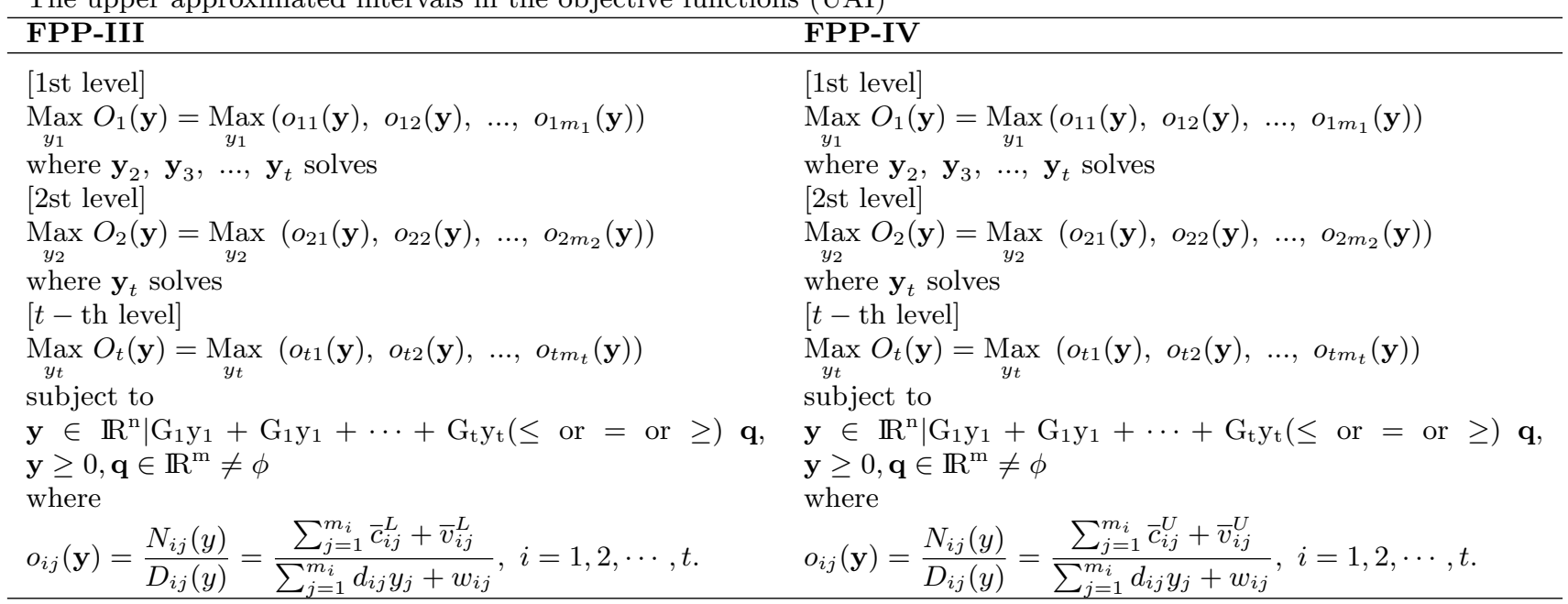

\section{Proposed Neutrosophic Fuzzy Goal Programming Approach}

The real-life complexity most often creates the indeterminacy situation or neutral thoughts while making optimal decisions. Apart from the acceptance and rejection degrees in the decision-making process, the indeterminacy degree also has much importance. Thus to cover the neutral thoughts or indeterminacy degree of the element into the feasible solution set, [31] investigated a neutrosophic set. The NS considers three sorts of membership functions, such as truth, indeterminacy, and falsity degrees into the feasible solution set. The idea of independent, neutral thoughts differs the NS with all the uncertain decision sets such as FS and IFS. The updated literature work solely highlights that many practitioners or researchers have shown the deep research keen in the neutrosophic field (see, $[6-8,13,29]$ ). The NS research domain would get exposure in the future and assist in dealing with indeterminacy degrees in neutrosophic uncertainty.

In ML-MOFPPs, if an imprecise aspiration level under the neutrosophic environment is assigned to each of the objectives at each level of the ML-MOFPPs, then such neutrosophic targets are termed as neutrosophic goals and dealt with neutrosophic decision-making techniques $[2,10]$. Hence the marginal evaluation of each 
neutrosophic target is characterized through truth, indeterminacy, and falsity membership functions, by eliciting the attainment of their respective satisfaction levels.

\subsection{Characterization of different membership functions under neutrosophic environment}

In multi-level decision-making problems, each DMs intend to minimize their objectives in each level over the same feasible region depicted by the system of constraints; hence the individual optimal solutions are obtained by them and can be regarded as the aspiration levels of their associated neutrosophic goals.

Assume that $\mathbf{y}^{i j}=\left(\mathbf{y}_{1}^{i j}, \mathbf{y}_{2}^{i j}, \ldots, \mathbf{y}_{t}^{i j}\right)$ and $o_{i j}^{\min }, i=1,2, \ldots, t, j=1,2, \ldots, m_{i}$ be the best individual optimal solutions of each DMs at each level respectively. Futhermore, consider that $l_{i j} \geq o_{i j}^{\min }$ denotes the aspiration level assigned to the $i j$-th objective $o_{i j}(\mathbf{y})$ (where $i j$ means that when $i=t$ for $t$-th level decision makers then $\left.j=1,2, \ldots, m_{i}\right)$. Moreover, also consider that $\mathbf{y}^{i *}=\left(\mathbf{y}_{1}^{i *}, \mathbf{y}_{2}^{i *}, \ldots, \mathbf{y}_{t}^{i *}\right), i=1,2, \ldots, t-1$, be the optimal solutions for the $t$-th level decision makers of ML-MOFPPs. Consequently, the neutrosophic goals of each objective function at each level and the vector-set of neutrosophic goals for the decision variables monitored by $t$-th level decision makers can be stated as follows:

$$
o_{i j}(\mathbf{y}) \tilde{<} l_{i j}, \quad i=1,2, \ldots, t, \quad j=1,2, \ldots, m_{i} \text { and } \mathbf{y}_{i} \stackrel{\sim}{=} \mathbf{y}_{i}^{i *}, \quad i=1,2, \ldots, t-1
$$

where $\tilde{<}$ and $\tilde{=}$ represents the degree of neutrosophy of the aspiration levels.

One can note that the solutions $\mathbf{y}^{i j}=\left(\mathbf{y}_{1}^{i j}, \mathbf{y}_{2}^{i j}, \ldots, \mathbf{y}_{t}^{i j}\right) ; i=1,2, \ldots, t, j=1,2, \ldots, m_{i}$ are probably different due to the conflicting nature of the objective functions at each level for all the decision makers. Therefore it can be obvious to consider that the values of $o_{g m}\left(\mathbf{y}_{1}^{g m}, \mathbf{y}_{2}^{g m}, \ldots, \mathbf{y}_{t}^{g m}\right) \geq o_{i j}^{\min } ; g=1,2, \ldots, t, m=1,2, \ldots, m_{i}$, and $\forall i j \neq g m$ with all values greater than $o_{g m}^{u}=\max \left[o_{i j}\left(\mathbf{y}_{1}^{g m}, \mathbf{y}_{2}^{g m}, \ldots, \mathbf{y}_{t}^{g m}\right) ; i=1,2, \ldots, t, j=1,2, \ldots, m_{i}\right.$ and $i j \neq g m]$ are absolutely unacceptable to the objective function $o_{g m}(\mathbf{y})=o_{g m}\left(\mathbf{y}_{1}, \mathbf{y}_{2}, \ldots, \mathbf{y}_{t}\right)$. As a result, $o_{g m}(\mathbf{y})$ can be taken as the upper tolerance limit $u_{g m}(\mathbf{y})$ of the neutrosophic goal to the objective functions. The upper and lower bounds for $i j$-th objective function under the neutrosophic environment can be obtained as follows:

$$
\begin{aligned}
& U_{i j}^{\mu}=u_{i j}, \quad L_{i j}^{\mu}=l_{i j} \\
& U_{i j}^{\lambda}=L_{i j}^{\mu}+a_{i j}, \quad L_{i j}^{\lambda}=L_{i j}^{\mu} \\
& U_{i j}^{\nu}=U_{i j}^{\mu}, \quad L_{i j}^{\nu}=L_{i j}^{\mu}+b_{i j}
\end{aligned}
$$

$$
\begin{array}{r}
\text { for truth membership } \\
\text { for indeterminacy membership } \\
\text { for falsity membership }
\end{array}
$$

where $a_{i j}$ and $b_{i j} \in(0,1)$ are predetermined real numbers.

Thus the different membership function namely; truth $\mu_{o_{i j}}\left(o_{i j}(\mathbf{y})\right)$, indeterminacy $\lambda_{o_{i j}}\left(o_{i j}(\mathbf{y})\right)$ and a falsity $\nu_{o_{i j}}\left(o_{i j}(\mathbf{y})\right)$ membership functions for the $i j$-th neutrosophic goals can be stated as follows:

$$
\begin{aligned}
& \mu_{o_{i j}}\left(o_{i j}(\mathbf{y})\right)=\left\{\begin{array}{lll}
0 & \text { if } & o_{i j}(\mathbf{y}) \leq L_{i j}^{\mu} \\
\frac{o_{i j}(\mathbf{y})-L_{i j}^{\mu}}{U_{i j}^{\mu}-L_{i j}^{\mu}} & \text { if } & L_{i j}^{\mu} \leq o_{i j}(\mathbf{y}) \leq U_{i j}^{\mu} \\
1 & \text { if } & o_{i j}(\mathbf{y}) \geq U_{i j}^{\mu}
\end{array}\right. \\
& \lambda_{o_{i j}}\left(o_{i j}(\mathbf{y})\right)=\left\{\begin{array}{lll}
0 & \text { if } & o_{i j}(\mathbf{y}) \leq L_{i j}^{\lambda} \\
\frac{o_{i j}(\mathbf{y})-L_{i j}^{\lambda}}{U_{i j}^{\lambda}-L_{i j}^{\lambda}} & \text { if } & L_{k}^{I} \leq o_{i j}(\mathbf{y}) \leq U_{i j}^{\lambda} \\
1 & \text { if } & o_{i j}(\mathbf{y}) \geq U_{i j}^{\lambda}
\end{array}\right. \\
& \nu_{o_{i j}}\left(o_{i j}(\mathbf{y})\right)=\left\{\begin{array}{lll}
0 & \text { if } & o_{i j}(\mathbf{y}) \geq U_{i j}^{\nu} \\
\frac{U_{i j}^{\nu}-o_{i j}(\mathbf{y})}{U_{i j}^{\nu}-L_{i j}^{\nu}} & \text { if } & L_{i j}^{\nu} \leq o_{i j}(\mathbf{y}) \leq U_{i j}^{\nu} \\
1 & \text { if } & o_{i j}(\mathbf{y}) \leq L_{i j}^{\nu}
\end{array}\right.
\end{aligned}
$$


To construct the different membership functions for the decision variables monitered by $i$-th decision makers, firstly the optimal solution for the $t$-th level MOFPPs, $\mathbf{y}^{i *}=\left(\mathbf{y}_{1}^{i *}, \mathbf{y}_{2}^{i *}, \ldots, \mathbf{y}_{t}^{i *}\right), i=1,2, \ldots, t-1$, should be carried out by using any appropriate method for MOFPPs optimization techniques.

In a neutrosophic decision environment, the neutrosophic goals comprising the decision-makers' objective functions at a different level and the neutrosophic goals of the decision variable vectors are monitored by leader $(t-1)$-th level decision-makers. The attainment degrees to their aspiration levels to the extent possible are virtually achieved by the eventual achievement of their respective memberships, namely; truth, indeterminacy, and falsity membership functions to their utmost degrees. This aspect of the neutrosophic fuzzy programming approach enables a neutrosophic fuzzy goal programming technique as a justified approach for solving the leader $t$-th level MOFPPs and consequently ML-MOFPPs.

\subsection{Neutrosophic Fuzzy Goal Programming}

In neutrosophic fuzzy goal programming approaches, each marginal evaluations is transformed into neutrosophic membership goals with respect to their highest attainment degrees. The maximum limit for attaining the truth membership function is unity (1). For the indeterminacy membership function, the highest attainment degree is half (0.5). Similarly, the falsity membership function can achieve the highest attainment degree zero $(0)$. Thus the converted neutrosophic membership goals is summarized as follows:

$$
\left.\begin{array}{l}
\mu_{o_{i j}}\left(o_{i j}(\mathbf{y})\right)+d_{i j \mu}^{-}-d_{i j \mu}^{+}=1, \\
\lambda_{o_{i j}}\left(o_{i j}(\mathbf{y})\right)+d_{i j \lambda}^{-}-d_{i j \lambda}^{+}=0.5, \\
\nu_{o_{i j}}\left(o_{i j}(\mathbf{y})\right)+d_{i j \nu}^{-}-d_{i j \nu}^{+}=0,
\end{array}\right\} \forall i=1,2, \ldots, t, \quad j=1,2, \ldots, m_{i}
$$

or equivalently represented as follows:

$$
\left.\begin{array}{l}
\frac{o_{i j}(\mathbf{y})-L_{i j}^{\mu}}{U_{i j}^{\mu}-L_{i j}^{\mu}}+d_{i j \mu}^{-}-d_{i j \mu}^{+}=1, \\
\frac{o_{i j}(\mathbf{y})-L_{i j}^{\lambda}}{U_{i j}^{\lambda}-L_{i j}^{\lambda}}+d_{i j \lambda}^{-}-d_{i j \lambda}^{+}=0.5, \\
\frac{U_{i j}^{\nu}-o_{i j}(\mathbf{y})}{U_{i j}^{\nu}-L_{i j}^{\nu}}+d_{i j \nu}^{-}-d_{i j \nu}^{+}=0,
\end{array}\right\} \forall i=1,2, \ldots, t, \quad j=1,2, \ldots, m_{i}
$$

where $d_{i j}^{-}, d_{i j}^{+} . \geq 0$; and $d_{i j .}^{-} \times d_{i j .}^{+}=0, \quad \forall i=1,2, \ldots, t-1, \quad k=1,2, \ldots, n_{i}$ are the over and under deviations for truth, indeterminacy and a falsity membership goals from their respective aspiration levels under neutrosophic environment.

In goal programming strategy, the over and/or under deviational variables vectors are considered in the objective function to minimize them and solely depends on the nature of objective function that are being optimized. In the proposed neutrosophic goal progrmming technique, the over deviational variables for neutrosophic goals of each objective function, $d_{i j}^{+} . \forall i=1,2, \ldots, t, j=1,2, \ldots, m_{i}$ are needed to be minimized to attain the neutrosophic fuzzy goals. Hence the proposed final solution model for ML-MOFPPs can be summarized as follows (4.6):

$$
\begin{aligned}
\text { Min } \mathrm{F}= & \sum_{j=1}^{m_{1}} w_{1 j \mu}^{+} d_{1 j \mu}^{+}+\sum_{j=1}^{m_{2}} w_{2 j \mu}^{+} d_{2 j \mu}^{+}+\cdots+\sum_{j=1}^{m_{t}} w_{t j \mu}^{+} d_{t j \mu}^{+}+\sum_{j=1}^{m_{1}} w_{1 j \lambda}^{+} d_{1 j \lambda}^{+}+\sum_{j=1}^{m_{2}} w_{2 j \lambda}^{+} d_{2 j \lambda}^{+} \\
& +\cdots+\sum_{j=1}^{m_{t}} w_{t j \lambda}^{+} d_{t j \lambda}^{+}-\sum_{j=1}^{m_{1}} w_{1 j \nu}^{+} d_{1 j \nu}^{-}-\sum_{j=1}^{m_{2}} w_{2 j \nu}^{+} d_{2 j \nu}^{-}-\cdots-\sum_{j=1}^{m_{t}} w_{t j \nu}^{+} d_{t j \nu}^{-}
\end{aligned}
$$

subject to

$$
\frac{o_{i j}(\mathbf{y})-L_{i j}^{\mu}}{U_{i j}^{\mu}-L_{i j}^{\mu}}+d_{t j \mu}^{-}-d_{t j \mu}^{+}=1, \quad i=1,2, \ldots, t, \quad j=1,2, \ldots, m_{i}
$$




$$
\begin{aligned}
& \frac{o_{i j}(\mathbf{y})-L_{i j}^{\lambda}}{U_{i j}^{\lambda}-L_{i j}^{\lambda}}+d_{t j \lambda}^{-}-d_{t j \lambda}^{+}=0.5, \quad i=1,2, \ldots, t, \quad j=1,2, \ldots, m_{i} \\
& \frac{U_{i j}^{\nu}-o_{i j}(\mathbf{y})}{U_{i j}^{\nu}-L_{i j}^{\nu}}+d_{t j \nu}^{-}-d_{t j \nu}^{+}=0, \quad i=1,2, \ldots, t, \quad j=1,2, \ldots, m_{i} \\
& G_{1} y_{1}+G_{1} y_{1}+\cdots+G_{t} y_{t}(\leq \text { or }=\text { or } \geq) \mathbf{q}, \mathbf{y} \geq 0 \\
& d_{i j .}^{-}, d_{i j .}^{+} \geq 0 \text { and } d_{i j}^{-} \times d_{i j .}^{+}=0, \quad \forall i=1,2, \ldots, t, \quad j=1,2, \ldots, m_{i} .
\end{aligned}
$$

The numerical weights $w_{i j}^{-}$state the relative importance of achieving the satisfactory levels of the respective neutrosophic goals. To assign the relative importance of the neutrosophic goals properly, the values of $w_{i j}^{-}$are determined as follows (4.14):

$$
w_{i j}^{-}=\frac{1}{u_{i j}-g_{i j}}, \quad \forall i=1,2, \ldots, t, \quad j=1,2, \ldots, m_{i} .
$$

The propounded NFGP procedures are based on a neutrosophic fuzzy decision set and applied to ML-MOFPPs. To formulate proposed NFGP models of the ML-MOFPPs, neutrosophic goals of the objectives are obtained by individual optimal solutions. The associated membership functions are then depicted for marginal evaluations of each objective function under the neutrosophic environment. These marginal evaluations are converted into neutrosophic flexible membership goals by employing over and under deviational variables and allowing the highest truth membership value (unity), indeterminacy value (half), and a falsity value (zero) as aspiration levels to each of them. To determine the membership functions of the constraints monitored by any level decision-maker, the optimal solution of the corresponding MOFPP is separately solved. A marginal relaxation of the decisions is prescribed to avoid decision deadlock. Finally, by tuning the weight parameters, one can get the optimal solutions for all levels in the hierarchical decision-making problems.

\subsection{Step-wise solution algorithms for ML-MOFPPs}

The proposed neutrosophic fuzzy goal programming (NFGP) algorithm for solving ML-MOFPPs is suggested under neutrosophic environment. The step-wise solution algorithm can be summarized as follows:

Step-1 Formulate the ML-MOFPPs with rough intervals co-efficients in the objective functions.

Step-2 Transform the ML-MOFPPs into lower and upper aproximated intervals as given in FPP-I, FPP-II, FPP-III and FPP-IV respectively.

Step-3 Compute $u_{i j}, g_{i j}, w_{i j}^{-}$for the problem FPP-I.

Step-4 Elicit the different membership functions under neutrosophic environment as given in Equations (4.1)(4.3).

Step-5 Repeat the same steps for solving FPP-II, FPP-III and FPP-IV.

Step-6 Construct the final solvable NFGP model (4.6) and solved using some suitable optimization software.

\section{Numerical illustrations}

The following numerical example, consisting of tri-level multiobjective interval fractional programming problems, is depicted to verify and validate the proposed NFGP optimization algorithms. The rough intervals for the various parameters are randomly generated using the statistical R-software. All the optimization models are coded in AMPL language and solution results are obtained by solver CONOPT available on NEOS server 5.0. The access is permitted through on-line medium by Wisconsin Institutes for Discovery, University of Wisconsin, 
Madison, See $[15,30]$.

[1st level]

$$
\begin{aligned}
\operatorname{Max}_{y_{1}} O_{1}(\mathbf{y})= & \operatorname{Max}_{11}(\mathbf{y})=\frac{2([2,3],[1,5]) y_{1}+([3,5],[2,7]) y_{2}+y_{3}+([2,3],[1,4])}{2 y_{1}+y_{2}+y_{3}+1} \\
& \operatorname{Max}_{12}(\mathbf{y})=\frac{([6,7],[5,9]) y_{1}-y_{2}+([1,3],[1,6]) y_{3}+([1,3],[0,5])}{y_{2}+y_{3}+3}
\end{aligned}
$$

where $\mathbf{y}_{2}$ and $\mathbf{y}_{3}$ solves

[2nd level]

$$
\begin{aligned}
\operatorname{Max}_{y_{1}} O_{2}(\mathbf{y})= & \operatorname{Max} o_{21}(\mathbf{y})=\frac{2 y_{1}+([5,6],[3,8]) y_{2}-2([0,3],[0,6]) y_{3}+([5,6],[3,7])}{y_{1}+y_{3}+4}, \\
& \operatorname{Max} o_{22}(\mathbf{y})=\frac{y_{1}-([3,4],[2,6]) y_{2}+([1,3],[1,7]) y_{3}+([3,4],[2,6])}{2 y_{1}+y_{3}+6}
\end{aligned}
$$

where $\mathbf{y}_{3}$ solves

[3rd level]

$$
\begin{array}{r}
\operatorname{Max}_{y_{1}} O_{3}(\mathbf{y})=\operatorname{Max} o_{31}(\mathbf{y})=\frac{([2,5],[1,8]) y_{1}-2 y_{2}+y_{3}+([4,5],[3,6])}{y_{3}+2}, \\
\operatorname{Max} o_{32}(\mathbf{y})=\frac{5 y_{1}+2([1,2],[1,4]) y_{2}-y_{3}+([6,7],[5,8])}{y_{1}+3 y_{2}+y_{3}+7}
\end{array}
$$

\begin{tabular}{|c|c|}
\hline FPP-I & FPP-II \\
\hline$[1$ st level $]$ & {$[1$ st level $]$} \\
\hline $\begin{array}{l}\operatorname{Max}_{y_{1}}\left(\frac{4 y_{1}+3 y_{2}+y_{3}+2}{2 y_{1}+y_{2}+y_{3}+1}, \frac{6 y_{1}-y_{2}+y_{3}+1}{y_{2}+y_{3}+3}\right) \\
\text { where } \mathbf{y}_{2}, \mathbf{y}_{3} \text { solves } \\
{[2 \text { nd level] }}\end{array}$ & $\begin{array}{l}\operatorname{Max}_{y_{1}}\left(\frac{6 y_{1}+5 y_{2}+y_{3}+2}{2 y_{1}+y_{2}+y_{3}+1}, \frac{7 y_{1}-y_{2}+3 y_{3}+3}{y_{2}+y_{3}+3}\right) \\
\text { where } \mathbf{y}_{2}, \mathbf{y}_{3} \text { solves } \\
{[2 \text { nd level] }}\end{array}$ \\
\hline $\begin{array}{l}\operatorname{Max}_{y_{2}}\left(\frac{2 y_{1}+5 y_{2}+5}{y_{1}+y_{3}+4}, \frac{y_{1}-3 y_{2}+y_{3}+3}{2 y_{1}+y_{3}+6}\right) \\
\text { where } \mathbf{y}_{3} \text { solves } \\
{[3 \text { rd level] }}\end{array}$ & $\begin{array}{l}\underset{y_{2}}{\operatorname{Max}}\left(\frac{2 y_{1}+6 y_{2}-6 y_{3}+6}{y_{1}+y_{3}+4}, \frac{y_{1}-4 y_{2}+3 y_{3}+4}{2 y_{1}+y_{3}+6}\right) \\
\text { where } \mathbf{y}_{3} \text { solves } \\
{[3 \text { rd level] }}\end{array}$ \\
\hline $\begin{array}{l}\operatorname{Max}_{y_{3}}\left(\frac{2 y_{1}-2 y_{2}+y_{3}+4}{y_{3}+2}, \frac{5 y_{1}+2 y_{2}-y_{3}+6}{y_{1}+3 y_{2}+y_{3}+7}\right) \\
\text { subject to }\end{array}$ & $\begin{array}{l}\operatorname{Max}_{y_{3}}\left(\frac{5 y_{1}-2 y_{2}+y_{3}+5}{y_{3}+2}, \frac{5 y_{1}+4 y_{2}-y_{3}+7}{y_{1}+3 y_{2}+y_{3}+7}\right) \\
\text { subject to }\end{array}$ \\
\hline $\begin{array}{l}3 y_{1}+5 y_{2}+y_{3} \leq 35, \quad 2 y_{1}-y_{2}+12 y_{3} \leq 20 \\
5 y_{2}+6 y_{3} \leq 16, \quad y_{1}, y_{2}, y_{3} \geq 0\end{array}$ & $\begin{array}{l}3 y_{1}+5 y_{2}+y_{3} \leq 35, \quad 2 y_{1}-y_{2}+12 y_{3} \leq 20 \\
5 y_{2}+6 y_{3} \leq 16, \quad y_{1}, y_{2}, \quad y_{3} \geq 0\end{array}$ \\
\hline
\end{tabular}

subject to

$$
\begin{aligned}
& 3 y_{1}+5 y_{2}+y_{3} \leq 35, \quad 2 y_{1}-y_{2}+12 y_{3} \leq 20 \\
& 5 y_{2}+6 y_{3} \leq 16, \quad y_{1}, y_{2}, y_{3} \geq 0
\end{aligned}
$$

For solving the example, it will be transformed into lower and upper intervals problems and can be stated as follows:

In Table 1, the best and worst values of each objective function is depicted for three heirarchical level in MOLPP. To apply the propounded NFGP techniques, the aspiration values can be regarded as the optimal results. 
The upper approximated intervals in the objective functions (UAI)

\begin{tabular}{|c|c|}
\hline FPP-III & FPP-IV \\
\hline [1st level] & [1st level] \\
\hline $\begin{array}{l}\underset{y_{1}}{\operatorname{Max}}\left(\frac{2 y_{1}+2 y_{2}+y_{3}+1}{2 y_{1}+y_{2}+y_{3}+1}, \frac{5 y_{1}-y_{2}+y_{3}+1}{y_{2}+y_{3}+3}\right) \\
\text { where } \mathbf{y}_{2}, \mathbf{y}_{3} \text { solves } \\
{[2 \text { nd level] }}\end{array}$ & $\begin{array}{l}\underset{y_{1}}{\operatorname{Max}}\left(\frac{10 y_{1}+7 y_{2}+y_{3}+4}{2 y_{1}+y_{2}+y_{3}+1}, \frac{9 y_{1}-y_{2}+6 y_{3}+5}{y_{2}+y_{3}+3}\right) \\
\text { where } \mathbf{y}_{2}, \mathbf{y}_{3} \text { solves } \\
{[2 \text { nd level }]}\end{array}$ \\
\hline $\begin{array}{l}\underset{y_{2}}{\operatorname{Max}}\left(\frac{2 y_{1}+3 y_{2}+3}{y_{1}+y_{3}+4}, \frac{y_{1}-2 y_{2}+y_{3}+2}{2 y_{1}+y_{3}+6}\right) \\
\text { where } \mathbf{y}_{3} \text { solves } \\
{[3 \text { rd level] }}\end{array}$ & $\begin{array}{l}\underset{y_{2}}{\operatorname{Max}}\left(\frac{2 y_{1}+8 y_{2}-12 y_{3}+7}{y_{1}+y_{3}+4}, \frac{y_{1}-6 y_{2}+7 y_{3}+6}{2 y_{1}+y_{3}+6}\right) \\
\text { where } \mathbf{y}_{3} \text { solves } \\
{[3 \text { rd level] }}\end{array}$ \\
\hline $\begin{array}{l}\operatorname{Max}_{y_{3}}\left(\frac{y_{1}-2 y_{2}+y_{3}+3}{y_{3}+2}, \frac{5 y_{1}+2 y_{2}-y_{3}+5}{y_{1}+3 y_{2}+y_{3}+7}\right) \\
\text { subject to } \\
3 y_{1}+5 y_{2}+y_{3} \leq 35, \quad 2 y_{1}-y_{2}+12 y_{3} \leq 20 \\
5 y_{2}+6 y_{3} \leq 16, \quad y_{1}, y_{2}, y_{3} \geq 0\end{array}$ & $\begin{array}{l}\operatorname{Max}_{y_{3}}\left(\frac{8 y_{1}-2 y_{2}+y_{3}+6}{y_{3}+2}, \frac{5 y_{1}+8 y_{2}-y_{3}+8}{y_{1}+3 y_{2}+y_{3}+7}\right) \\
\text { subject to } \\
3 y_{1}+5 y_{2}+y_{3} \leq 35, \quad 2 y_{1}-y_{2}+12 y_{3} \leq 20 \\
5 y_{2}+6 y_{3} \leq 16, \quad y_{1}, y_{2}, y_{3} \geq 0\end{array}$ \\
\hline
\end{tabular}

TABLE 1. Individual minimum and maximum values for each objectives

\begin{tabular}{llrrrrl}
\hline \hline & $o_{11}(y)$ & $o_{12}(y)$ & \multicolumn{1}{c}{$o_{21}(y)$} & \multicolumn{1}{c}{$o_{22}(y)$} & \multicolumn{1}{c}{$o_{31}(y)$} & \multicolumn{2}{c}{$o_{32}(y)$} \\
\cline { 2 - 7 } $\max _{S} o_{i j}(y)$ & 2.76 & 20.33 & 05.25 & 0.61 & 12 & 3.29 \\
$\min _{S} o_{i j}(y)$ & 1.37 & -0.35 & 0.88 & -1.10 & 0.40 & 0.50 \\
$u_{i j}(y)$ & 2.70 & 20.00 & 5.00 & 0.60 & 12.00 & 3.20 \\
$g_{i j}(y)$ & 1.30 & -0.35 & 0.88 & -1.00 & 0.40 & 0.50 \\
$w_{i j}(y)$ & 0.71 & 0.09 & 0.24 & 0.62 & 0.08 & 0.37 \\
\hline
\end{tabular}

1st level decision-maker's NFGP model:

$$
\begin{aligned}
& \text { Min F }=0.714 d_{11 \mu}^{-}+0.094 d_{12 \mu}^{-}+0.714 d_{11 \lambda}^{-}+0.094 d_{12 \lambda}^{-}-0.714 d_{11 \nu}^{+}-0.094 d_{12 \nu}^{+} \\
& \text {subject to } \\
& -y_{1}+0.214 y_{2}-1.214 y_{3}+d_{11 \mu}^{-}-d_{11 \mu}^{+}=0.50 \\
& -y_{1}+0.214 y_{2}-1.214 y_{3}+d_{11 \lambda}^{-}-d_{11 \lambda}^{+}=0.143 \\
& -y_{1}+0.214 y_{2}-1.214 y_{3}+d_{11 \nu}^{-}-d_{11 \nu}^{+}=0.03 \\
& -2 y_{1}-y_{2}-y_{3}+d_{11 \mu}^{-} \leq 1.00 \\
& -2 y_{1}-y_{2}-y_{3}+d_{11 \lambda}^{-} \geq 0.81 \\
& -2 y_{1}-y_{2}-y_{3}+d_{11 \nu}^{-} \geq 1.27 \\
& 0.294 y_{1}-1.029 y_{2}-0.931 y_{3}+d_{12 \mu}^{-}-d_{12 \mu}^{+}=2.891 \\
& 0.294 y_{1}-1.029 y_{2}-0.931 y_{3}+d_{12 \lambda}^{-}-d_{12 \lambda}^{+}=1.201 \\
& 0.294 y_{1}-1.029 y_{2}-0.931 y_{3}+d_{12 \nu}^{-}-d_{12 \nu}^{+}=1.597 \\
& -y_{2}-y_{3}+d_{12 \mu}^{-} \leq 3 \\
& -y_{2}-y_{3}+d_{12 \lambda}^{-} \geq 2.54 \\
& -y_{2}-y_{3}+d_{12 \nu}^{-} \geq 1.96 \\
& 3 y_{1}+5 y_{2}+y_{3} \leq 35, \quad 2 y_{1}-y_{2}+12 y_{3} \leq 20, \\
& 5 y_{2}+6 y_{3} \leq 16, \quad y_{1}, y_{2}, y_{3} \geq 0, \\
& d_{i j .}^{-}, d_{i j .}^{+} \geq 0 \text { and } d_{i j .}^{-} \times d_{i j .}^{+}=0, \quad \forall i=1, \quad j=1,2 .
\end{aligned}
$$

With the help of optimizing software, the optimal solution of the problem given in Equation (5.1) are $\mathbf{y}^{1 *}=(0,2.33,0)$. 
2nd level decision maker's NFGP model:

Min $\mathrm{F}=0.714 d_{11 \mu}^{-}+0.094 d_{12 \mu}^{-}+0.714 d_{11 \lambda}^{-}+0.094 d_{12 \lambda}^{-}-0.714 d_{11 \nu}^{+}-0.094 d_{12 \nu}^{+}$

$$
+0.243 d_{21 \mu}^{-}+0.625 d_{22 \mu}^{-}+0.243 d_{21 \lambda}^{-}+0.625 d_{22 \lambda}^{-}-0.243 d_{21 \nu}^{+}-0.625 d_{22 \nu}^{+}
$$

subject to

$$
\begin{aligned}
& -0.728 y_{1}+0.214 y_{2}-0.001 y_{3}+d_{21 \mu}^{-}-d_{21 \mu}^{+}=3.641 \\
& -0.728 y_{1}+0.214 y_{2}-0.001 y_{3}+d_{21 \lambda}^{-}-d_{21 \lambda}^{+}=2.654 \\
& -0.728 y_{1}+0.214 y_{2}-0.001 y_{3}+d_{21 \nu}^{-}-d_{21 \nu}^{+}=2.145 \\
& -y_{1}-y_{3}+d_{21 \mu}^{-} \leq 4.00 \\
& -y_{1}-y_{3}+d_{21 \lambda}^{-} \geq 3.29 \\
& -y_{1}-y_{3}+d_{21 \nu}^{-} \geq 2.64 \\
& -1.25 y_{1}-1.875 y_{2}-2.25 y_{3}+d_{22 \mu}^{-}-d_{22 \mu}^{+}=0.375 \\
& -1.25 y_{1}-1.875 y_{2}-2.25 y_{3}+d_{22 \lambda}^{-}-d_{22 \lambda}^{+}=0.684 \\
& -1.25 y_{1}-1.875 y_{2}-2.25 y_{3}+d_{22 \nu}^{-}-d_{22 \nu}^{+}=0.369 \\
& -2 y_{1}-y_{3}+d_{22 \mu}^{-} \leq 6.00 \\
& -2 y_{1}-y_{3}+d_{22 \lambda}^{-} \geq 5.31 \\
& -2 y_{1}-y_{3}+d_{22 \nu}^{-} \geq 4.09 \\
& y_{1}=0, \quad y_{2}, y_{3} \geq 0, \\
& \text { constraints }(5.1) \\
& d_{i j .}^{-}, d_{i j .}^{+} \geq 0 \text { and } d_{i j .}^{-} \times d_{i j .}^{+}=0, \quad \forall i=1, \quad j=1,2 .
\end{aligned}
$$

The optimal solution for the second level NFGP model in Equation (5.2) is obtained as $\mathbf{y}^{2 *}=(0.31,0.00,0.03)$.

$\underline{\text { 3rd level decision maker's NFGP model: }}$

Min $\mathrm{F}=0.714 d_{11 \mu}^{-}+0.094 d_{12 \mu}^{-}+0.714 d_{11 \lambda}^{-}+0.094 d_{12 \lambda}^{-}-0.714 d_{11 \nu}^{+}-0.094 d_{12 \nu}^{+}$

$$
+0.243 d_{21 \mu}^{-}+0.625 d_{22 \mu}^{-}+0.243 d_{21 \lambda}^{-}+0.625 d_{22 \lambda}^{-}-0.243 d_{21 \nu}^{+}-0.625 d_{22 \nu}^{+}
$$

$$
+0.086 d_{31 \mu}^{-}+0.370 d_{32 \mu}^{-}+0.086 d_{31 \lambda}^{-}+0.370 d_{32 \lambda}^{-}-0.086 d_{31 \nu}^{+}-0.370 d_{32 \nu}^{+}
$$

subject to

$$
\begin{aligned}
& 0.172 y_{1}-0.172 y_{2}-0.948 y_{3}+d_{31 \mu}^{-}-d_{31 \mu}^{+}=1.724 \\
& 0.172 y_{1}-0.172 y_{2}-0.948 y_{3}+d_{31 \lambda}^{-}-d_{31 \lambda}^{+}=1.312 \\
& 0.172 y_{1}-0.172 y_{2}-0.948 y_{3}+d_{31 \nu}^{-}-d_{31 \nu}^{+}=0.215 \\
& -y_{3}+d_{31 \mu}^{-} \leq 2.00 \\
& -y_{3}+d_{31 \lambda}^{-} \geq 1.62 \\
& -y_{3}+d_{31 \nu}^{-} \geq 1.94 \\
& 0.665 y_{1}-2.815 y_{2}-1.555 y_{3}+d_{32 \mu}^{-}-d_{32 \mu}^{+}=0.375 \\
& 0.665 y_{1}-2.815 y_{2}-1.555 y_{3}+d_{32 \lambda}^{-}-d_{32 \lambda}^{+}=0.684
\end{aligned}
$$


TABLE 2. Comparision of optimal solutions

\begin{tabular}{|c|c|c|}
\hline \multirow[t]{2}{*}{ The surely optimal interval } & \multicolumn{2}{|c|}{ The possibly optimal interval } \\
\hline & Proposed NFGP Algorithm & [24] Approach \\
\hline \multicolumn{3}{|l|}{ [1st level] } \\
\hline$\left[\underline{o}_{11}^{L}, \underline{Q}_{11}^{U}\right]=[2.009,3]$ & {$[1.1901,3.961]$} & {$[1.183,4]$} \\
\hline$\left[\underline{o}_{12}^{L}, \underline{o}_{12}^{U}\right]=[0.334,1]$ & {$[-0.0434,1.592]$} & {$[-0.0577,1.667]$} \\
\hline \multicolumn{3}{|l|}{$[2$ nd level $]$} \\
\hline$\left[\underline{o}_{21}^{L}, \underline{o}_{21}^{U}\right]=[1.25,1.5]$ & {$[0.9124,1.682]$} & {$[0.887,1.75]$} \\
\hline$\left[\underline{o}_{22}^{L}, \underline{o}_{22}^{U}\right]=[0.499,0.667]$ & {$[0.2934,0.9823]$} & {$[0.272,1]$} \\
\hline \multicolumn{3}{|l|}{ [3rd level] } \\
\hline$\left[\underline{o}_{31}^{L}, \underline{o}_{31}^{U}\right]=[2.003,2.50]$ & {$[1.3081,2.9632]$} & {$[1.316,3]$} \\
\hline$\left[\underline{o}_{32}^{L}, \underline{o}_{32}^{U}\right]=[0.858,1]$ & {$[0.7731,1.1231]$} & {$[0.71,1.14]$} \\
\hline
\end{tabular}

TABLE 3. Theoritical comparision of proposed NFGP algorithms with others

\begin{abstract}
Proposed NFGP Approach
Proposed approach considers the indeterminacy degree in decision-making process.

The overall satisfactory degree is achieved by attaining the neutrosophic fuzzy goals.

It characterizes neutrosophic membership functions for both objectives as well as constraints under neutrosophic environment.
\end{abstract}

Additional predetermined parameters in indeterminacy and falsity degrees make the decisions more flexible according to decision makers' choices.

\section{Other Approaches}

$[18,22-24]$ and [21] cannot deal with indeterminacy in decision-making processes.

In [18, 22-24] and [21] approaches, satisfactory degree is acheived by attaining the fuzzy goals.

$[18,22-24]$ and $[21]$ do not cover this aspects.

This facility is not provided in [18,22-24] and [21].

$$
\begin{aligned}
& 0.665 y_{1}-2.815 y_{2}-1.555 y_{3}+d_{32 \nu}^{-}-d_{32 \nu}^{+}=0.369 \\
& -y_{1}-3 y_{2}-y_{3}+d_{32 \mu}^{-} \leq 7.00 \\
& -y_{1}-3 y_{2}-y_{3}+d_{32 \lambda}^{-} \geq 6.58 \\
& -y_{1}-3 y_{2}-y_{3}+d_{32 \nu}^{-} \geq 5.41 \\
& y_{1}=0, y_{2}=0, \quad y_{3} \geq 0, \\
& \text { constraints }(5.2) \\
& d_{i j .}^{-}, d_{i j .}^{+} \geq 0 \text { and } d_{i j .}^{-} \times d_{i j .}^{+}=0, \quad \forall i=1, \quad j=1,2 .
\end{aligned}
$$

The final optimal solutions for the ML-MOFPPs given in Equation (5.22) is obtained as $\mathbf{y}^{3 *}=$ $(0.005,0.003,0.000)$ with the different objectives values $o_{11}=2.009, o_{12}=0.334, o_{21}=1.250, o_{22}=0.499$, $o_{31}=2.003$ and $o_{32}=0.858$ respectively. Similarly, on implementing the proposed NFGP algorithm, we get FPPII, FPP-III and FPP-IV in the specified intervals. A comparative study is performed between the proposed NFGP algorithm and [24] presented in the Table 2 . From Table 2 , it can be observed that the possibly optimal range obtained by proposed NFGP algorithm is quite narrow (shorter) than [24] approah. This shows that the possibility of getting an optimal solution within short specified intervals is very high as compared to longer one. Moreover, the theoritical contributions in the domain of ML-MOFPPs is also summarized in Table 3. 
From the managerial perspective, the proposed techniques intensely express neutral thoughts during hierarchical decision-making processes. Rough intervals are valuable and new tools to tackle the uncertainty in decision-making problems. The method proposed in this paper helps to solve ML-MOFPPs with rough interval parameters where the DMs present in various levels usually have conflicting objectives. However, there exists no other method in the literature for solving ML-MOFPPs with the neutrosophic fuzzy concept in the neutrosophic fuzzy goals. This is the first approach in the neutrosophic fuzzy environment with rough interval parameters and a weighting scheme. Neutrosophic goal achievement functions are constructed to attain the three different targeted goals. Since in an ML-MOFPPs, a decision deadlock situation may arise due to conflicting objectives, each level DM provides preference bounds on the decision variables controlled by him/her. GP strategy is then employed to obtain the optimal compromise solution of the ML-MOFPPs with rough interval parameters. The method discussed here can be applied in decision-making in large hierarchical organizations where multiple DMs have conflicting objectives. The actual decision-making process can be better represented through the neutrosophic fuzzy concept as it considers all three different aspects of decision making, i.e., truth, falsity, and indeterminacy. Here indeterminacy/neutral is considered as an independent factor that has a crucial role in decision making. The novelty of the method lies in its simplicity and efficient handling of indeterminate data. At the managerial level, each DMs can express their indeterminate degrees independently, making the proposed approach more efficient and flexible, which is also much closer to reality. The neutrosophic goal programming strategy discussed here can be helpful to solve real problems in a vast range of fields, including agriculture, transportation, biofuel production, etc., in a neutrosophic environment.

\section{Conclusions}

This paper proposes neutrosophic fuzzy goal programming algorithms for the solutions of ML-MOFPPs. The neutrosophic fuzzy goal programming is constructed to minimize the group tolerance of a satisfactory degrees, and to attain the highest degree for truth (unity), indeterminacy (half) and a falsity (zero) of each kind of the prescribed membership functions goals to the utmost possible by minimizing their respective deviational variables and so that obtain the optimal solutions. The prime advantage of the proposed neutrosophic fuzzy goal programming algorithms is the chances of refusing the solution repeatedly by the leader-level decision-maker and re-evaluation of the problem again and again by restating the defined membership functions required to reach the optimal solution would not arise.

The NFGP algorithm considers the different neutrosophic goals as well as the different membership functions for the neutrosophic goals for the decision variable vectors at each level except the follower level of the MLMOFPPs. It also solves the MOFPPs of ML-MOFPPs by taking into account the decisions of MOFPPs for the leader level only. A numerical example is shown to verify and validate the proposed NFGP algorithms. The degree of indeterminacy may arise in the hierarchical decision-making processes and can be overcome by utilizing the proposed algorithms.

In the future, multi-level multi-objective integer fractional programming problems, multi-level multi-objective mixed integer fractional decision-making problems, multi-level multi-objective integer quadratic programming with rough parameters in the objective functions; in the constraints and both would be studied. It can also be implemented in real-life applications such as transportation, assignment, vendor selection, inventory control optimization, supply chain planning, etc., problems in multi-level decision-making scenarios. Also, the proposed work can be extended with different forms of parameters such as fuzzy, stochastic, and uncertain instead of taking the rough intervals, and the comparative study would be made. Studying more properties of the introduced models can be a subject for further research. Moreover, applying the new models to deal with real-world problems is an attractive area for research.

Acknowledgements. Authors are overwhelmed to the Editor-in-Chief, anonymous Guest Editor, and potential reviewers for providing in-depth advices. The authors extend their appreciation to King Saud University for funding this work through Researchers Supporting Project number (RSP-2021/387), King Saud University, Riyadh, Saudi Arabia. 
Funding. This research work is supported through Researchers Supporting Project number (RSP-2021/387), King Saud University, Riyadh, Saudi Arabia.

Conflict of Interest. All authors declare no conflict of interest.

\section{REFERENCES}

[1] M.A. Abo-Sinna and I.A. Baky, Fuzzy goal programming procedure to bilevel multiobjective linear fractional programming problems. Int. J. Math. Math. Sci. 2010 (2010).

[2] A.Y. Adhami and F. Ahmad, Interactive pythagorean-hesitant fuzzy computational algorithm for multiobjective transportation problem under uncertainty. Int. J. Manag. Sci. Eng. Manag. 15 (2020) 1-10.

[3] F. Ahmad, Interactive neutrosophic optimization technique for multiobjective programming problems: an application to pharmaceutical supply chain management. Ann. Oper. Res. (2021a) 1-35.

[4] F. Ahmad, Robust neutrosophic programming approach for solving intuitionistic fuzzy multiobjective optimization problems. Complex Intell. Syst. (2021b) 1-20.

[5] F. Ahmad and A.Y. Adhami, Neutrosophic programming approach to multiobjective nonlinear transportation problem with fuzzy parameters. Int. J. Manag. Sci. Eng. Manag. 14 (2019a) 218-229.

[6] F. Ahmad and A.Y. Adhami, Total cost measures with probabilistic cost function under varying supply and demand in transportation problem. Opsearch 56 (2019b) 583-602.

[7] F. Ahmad, A.Y. Adhami and F. Smarandache, Single Valued Neutrosophic Hesitant Fuzzy Computational Algorithm for Multiobjective Nonlinear Optimization Problem. Neutrosophic Sets Syst. 22 (2018) 76-86.

[8] F. Ahmad, A.Y. Adhami and F. Smarandache, Neutrosophic optimization model and computational algorithm for optimal shale gas water management under uncertainty. Symmetry 11 (2019).

[9] F. Ahmad, A.Y. Adhami and F. Smarandache, Modified neutrosophic fuzzy optimization model for optimal closed-loop supply chain management under uncertainty. In Optimization theory based on neutrosophic and plithogenic sets, Elsevier (2020) 343-403.

[10] F. Ahmad, S. Ahmad and M. Zaindin, A sustainable production and waste management policies for covid-19 medical equipment under uncertainty: A case study analysis. Comput. Ind. Eng. 157 (2021) 107381.

[11] S. Ahmad, F. Ahmad and M. Sharaf, Supplier selection problem with type-2 fuzzy parameters: A neutrosophic optimization approach. Int. J. Fuzzy Syst. 23 (2021) 755-775.

[12] A.A.H. Ahmadini and F. Ahmad, A novel intuitionistic fuzzy preference relations for multiobjective goal programming problems. Int. J. Fuzzy Syst. 40 (2021a) 4761-4777.

[13] A.A.H. Ahmadini and F. Ahmad, Solving intuitionistic fuzzy multiobjective linear programming problem under neutrosophic environment. AIMS Math. 6 (2021b) 4556-4580.

[14] M. Arabani, Application of rough set theory as a new approach to simplify dams location. Sci. Iran. 13 (2006).

[15] E. Dolan, The neos server 4.0 administrative guide. Tech. Technical report, Memorandum ANL/MCS-TM-250, Mathematics and Computer Science Division, Argonne National Laboratory, Argonne, IL, USA (2001).

[16] A. Hamzehee, M.A. Yaghoobi and M. Mashinchi, Linear programming with rough interval coefficients. J. Intell. Fuzzy Syst. 26 (2014) 1179-1189.

[17] M. Imran, M.H. Agha, W. Ahmed, B. Sarkar and M.B. Ramzan, Simultaneous customers and supplier's prioritization: An ahp-based fuzzy inference decision support system (ahp-fidss). Int. J. Fuzzy Syst. 22 (2020) 2625-2651.

[18] K. Lachhwani and M.P. Poonia, Mathematical solution of multilevel fractional programming problem with fuzzy goal programming approach. J. Ind. Eng. Int. 8 (2012) 16.

[19] A.S. Mahapatra, B. Sarkar, M.S. Mahapatra, H.N. Soni and S.K. Mazumder, Development of a fuzzy economic order quantity model of deteriorating items with promotional effort and learning in fuzziness with a finite time horizon. Inventions 4 (2019) 36.

[20] A.I. Malik and B. Sarkar, Coordinating supply-chain management under stochastic fuzzy environment and lead-time reduction. Mathematics 7 (2019) 480.

[21] S. Mishra, Weighting method for bi-level linear fractional programming problems. Eur. J. Oper. Res. 183 (2007) $296-302$.

[22] S. Nayak and A. Ojha, On multi-level multi-objective linear fractional programming problem with interval parameters. RAIROOperations Research 53 (2019) 1601-1616.

[23] M. Osman, O. Emam and M. El Sayed, Solving multi-level multi-objective fractional programming problems with fuzzy demands via fgp approach. Int. J. Appl. Comput. Math. 4 (2018) 41.

[24] M.S. Osman, K.R. Raslan, O.E. Emam and F.A. Farahat, Solving multi-level multi-objective fractional programming problem with rough intervals in the objective functions. J. adv. math. Comput. Sci. (2017) 1-17.

[25] Z. Pawlak, Rough sets. Int. J. Comput. Inf. Syst. 11 (1982) 341-356.

[26] Z. Pawlak and A. Skowron, Rudiments of rough sets. Inf. Sci. 177 (2007) 3-27.

[27] S. Pramanik and T.K. Roy, Fuzzy goal programming approach to multilevel programming problems. Eur. J. Oper. Res. 176 (2007) 1151-1166.

[28] H. Rashmanlou, M. Pal, S. Raut, F. Mofidnakhaei and B. Sarkar, Novel concepts in intuitionistic fuzzy graphs with application. J. Intell. Fuzzy Syst. 37 (2019) 3743-3749. 
[29] R.M. Rizk-Allah, A.E. Hassanien and M. Elhoseny, A multi-objective transportation model under neutrosophic environment. Comput. Electr. Eng. 69 (2018) 705-719.

[30] N. Server, State-of-the-Art Solvers for Numerical Optimization (2016).

[31] F. Smarandache, A unifying field in logics: Neutrosophic logic. In Philosophy, American Research Press (1999) 1-141.

\section{Subscribe to Open (S2O) A fair and sustainable open access model}

This journal is currently published in open access with no charge for authors under a Subscribe-to-Open model (S2O). Open access is the free, immediate, online availability of research articles combined with the rights to use these articles fully in the digital environment.

$\mathrm{S} 2 \mathrm{O}$ is one of the transformative models that aim to move subscription journals to open access. Every year, as long as the minimum amount of subscriptions necessary to sustain the publication of the journal is attained, the content for the year is published in open access.

\section{Ask your library to support open access by subscribing to this S2O journal.}

Please help to maintain this journal in open access! Encourage your library to subscribe or verify its subscription by contacting subscribers@edpsciences.org

We are thankful to our subscribers and sponsors for making it possible to publish the journal in open access, free of charge for authors. More information and list of sponsors: https://www.edpsciences.org/en/maths-s2o-programme 